\title{
Estimates of genetic parameters for total milk yield over multiple ages in Brazilian Murrah buffaloes using different models
}

\author{
R.C. Sesana ${ }^{1}$, F. Baldi ${ }^{1}$, R.R.A. Borquis ${ }^{1}$, A.B. Bignardi ${ }^{1}$, \\ N.A. Hurtado-Lugo ${ }^{1}$, L. El Faro ${ }^{2}$, L.G. Albuquerque ${ }^{1,3}$ and H. Tonhati ${ }^{1,3}$ \\ ${ }^{1}$ Departamento de Zootecnia, Universidade Estadual Paulista, \\ Jaboticabal, SP, Brasil \\ ${ }^{2}$ Agência Paulista de Tecnologia dos Agronegócios, \\ Pólo Regional Centro Leste, Ribeirão Preto, SP, Brasil \\ ${ }^{3}$ Instituto Nacional de Ciência e Tecnologia - Ciência Animal, \\ Universidade Federal de Viçosa, Viçosa, MG, Brasil \\ Corresponding author: H. Tonhati \\ E-mail: tonhati@fcav.unesp.br
}

Genet. Mol. Res. 13 (2): 2784-2795 (2014)

Received February 28, 2013

Accepted September 19, 2013

Published April 14, 2014

DOI http://dx.doi.org/10.4238/2014.April.14.7

\begin{abstract}
The objective of this study was to estimate variance components and genetic parameters for accumulated 305-day milk yield (MY305) over multiple ages, from 24 to 120 months of age, applying random regression (RRM), repeatability (REP) and multi-trait (MT) models. A total of 4472 lactation records from 1882 buffaloes of the Murrah breed were utilized. The contemporary group (herd-yearcalving season) and number of milkings (two levels) were considered as fixed effects in all models. For REP and RRM, additive genetic, permanent environmental and residual effects were included as random effects. MT considered the same random effects as did REP and RRM with the exception of permanent environmental effect. Residual variances were modeled by a step function with 1,4 , and 6 classes.
\end{abstract}


The heritabilities estimated with RRM increased with age, ranging from 0.19 to 0.34 , and were slightly higher than that obtained with the REP model. For the MT model, heritability estimates ranged from 0.20 (37 months of age) to 0.32 (94 months of age). The genetic correlation estimates for MY305 obtained by RRM (L23.res4) and MT models were very similar, and varied from 0.77 to 0.99 and from 0.77 to 0.99 , respectively. The rank correlation between breeding values for MY305 at different ages predicted by REP, MT, and RRM were high. It seems that a linear and quadratic Legendre polynomial to model the additive genetic and animal permanent environmental effects, respectively, may be sufficient to explain more parsimoniously the changes in MY305 genetic variation with age.

Key words: Bubalus bubalis; Random regression models; Heritability; Genetic correlation; Sire rank

\section{INTRODUCTION}

The Brazilian buffalo is one of the largest buffalo herds in the world with 1.2 million head. In the last decades, Brazilian buffalo milk yield per lactation has significantly increased, as a consequence of improvement in management, infrastructure, and feeding practices on buffalo farms (Vasconcellos and Tonhati, 1998; Tonhati et al., 2000a; Tonhati et al., 2008). However, to develop a buffalo breeding program, it is necessary to define appropriate models for genetic evaluation of total milk yield.

Several studies in dairy cattle have proposed the repeatability (REP) and multi-trait (MT) models to obtain variance components and genetic parameters for accumulated 305-day milk yield at multiple parities (Suzuki and VanVleck, 1994; García-Cortés et al., 1995; Powell and Norman, 2006). With the MT models, total milk yield records from different lactations are assumed as different traits and an unstructured covariance matrix may be used with the number of traits equal to the number of different measurements. This may result in a high over-parameterized model and high computational demands (Meyer and Hill, 1997). Alternatively, the REP model is less complex than the MT model, but it assumes that total milk yield records from different lactations are repetitions of the same trait, with constant genetic and non-genetic variances. According to Guo et al. (2002), REP does not fit the data well when later lactations are included in the analysis.

Recently, random regression models (RRM) have been proposed as an alternative for the analysis of longitudinal data or repeated measures records (Meyer, 2004; Schaeffer, 2004; Bignardi et al., 2009). RRM have been applied to model the population mean trend and random effects for first lactation test-day milk records (Breda et al., 2010; Sesana et al., 2010) and for 305-day milk yield (MY305) records over multiple parities (Guo et al., 2002). By applying RRM, the covariance functions that give the covariance between any two ages or lactations, within the range of age in the data, can be estimated and impose a structure on the covariance matrices (Meyer, 1998). Also, RRM allows changes in variance along the trajectory, providing estimates of breeding values for the entire trajectory with higher accuracies than the conventional MT model (Tier and Meyer, 2004; Boligon et al., 2011). 
In the literature, there are few works reporting genetic parameter estimates for buffalo total milk yield. According to Rosati and Van Vleck (2002), the majority of studies in buffaloes for productive traits have been conducted in Pakistan and India. In Egypt, Mourad and Mohamed (1995) and in Italy, Rosati and Van Vleck (2002), obtained heritability estimates for buffaloes total milk yield at different calvings. Recently, in Brazil, Tonhati et al. (2008) worked with dairy buffaloes from first to sixth lactation order and estimated genetic parameters for total milk yield and MY305.

In Brazil, the genetic evaluation for dairy buffaloes is carried out for total milk yield (MY305) using a REP model. This model allows the inclusion of all animal information available and few assumptions about the model. Recently, RRM are currently being used for national genetic evaluations of dairy cattle in several countries. To date, there is no consensus for buffalo milk yield about the most appropriate model for genetic evaluation of MY305. Thus, it is crucial to develop alternative models to be implemented in a genetic evaluation program for dairy buffaloes in Brazil. The objectives of this study were to estimate (co)variance components and genetic parameters for MY305 at different ages using REP, MT, and RRM models. Also, the breeding values for MY305 at different ages predicted from different models were compared.

\section{MATERIAL AND METHODS}

Buffaloes' milk yield records were obtained from monthly test-day records from the database of the Animal Science Department/FCAV, São Paulo State University. Data were from 12 herds located in the State of São Paulo, Brazil, and recorded from 1985 to 2005. The data set comprised 4472 complete lactation records from 1882 animals of the Murrah breed raised on pastures with feed supplementation during the dry period from April to September.

Lactation records were truncated at 305 days, since only $16 \%$ of records had shown lactation beyond this point. The data sets included only lactations where the first test-day milk record was measured from 5th to 45th day after calving. Lactation records less than 90 days long were excluded. Age of cows at calving ranged from 2 to 10 years.

Contemporary groups (CG) were defined as animals that calved in the same herd, year and season (season $1=$ October to March; season $2=$ April to September). CG with less than 3 lactations and animals with milk yield above or below 3.0 standard deviations from CG mean, were excluded from the data set. The observations were grouped into classes of age at calving (in months) from 24 to 120 months of age.

In the MT model, five traits were considered as different traits, MY305 at 37, 52, 66, 79 , and 94 months of age (calving age). The number of MY305 records (means \pm standard deviation for MY305) at 37, 52, 66, 79, and 94 months of age were $1496(1599 \pm 571) ; 1050$ (1804 \pm 674$) ; 769$ (1881 \pm 719$) ; 503$ (1936 \pm 756$)$; and 402 (1969 \pm 750$)$, respectively. The MT model included the additive genetic and residual as random effects, and the CG and number of milkings per day (two levels) were included as fixed effects. The genetic additive, residual and permanent environmental effects were included as random effects in the REP and RRM models. The same fixed effects considered in the MT model were included in the REP model. The age of cow at calving (in months) was included as a covariable (linear and quadratic effects) in the REP model. There were 2810 animals and 261 sires in the relation- 
ship matrix. The matrix representation of the MT and REP models is:

$$
\mathrm{y}=\mathrm{Xb}+\mathrm{Za}+\mathrm{Wc}+\mathrm{e}
$$

where, $y$ is a vector of observed traits; $X$ is the incidence matrix of fixed effects; $b$ is a vector of fixed effects; $Z$ is the incidence matrix of additive genetic random effects; $a$ is a vector of additive genetic random effects; $W$ is the incidence matrix of permanent environmental random effect (only for REP); $c$ is a vector of permanent environmental random effects (only for REP); and $e$ is a vector of random error effects. The assumptions about expectation and variances are: $\mathrm{E}[\mathrm{y}]=\mathrm{Xb}$; $\operatorname{Var}(\mathrm{a})=\mathrm{A}$ Sa; $\operatorname{Var}(\mathrm{c})=\mathrm{I} \mathrm{Sc}$; and $\operatorname{Var}(\mathrm{e})=\mathrm{I} \mathrm{Se}$. Sa is the additive genetic effect covariance matrix; $\mathrm{Sc}$ is the permanent environmental effect covariance matrix and $\mathrm{Se}$ is the residual covariance matrix; A is the relationship matrix between animals; I is the identity matrix; and $\otimes$ is the Kroenecker product between matrices.

A total of 9 random regression models were fitted with different residual variance structures and order of covariance function for additive genetic and permanent environmental effects. Residual variances were modeled by step function with 1, 4 (24-47, 48-71, 72-95 and 96-120 months of age) and 6 (24-40, 41-56, 57-63, 64-72, 73-96, and 97-120 months of age) classes. The random regression analyses were carried out using a one-trait model. The GC and the population mean trend, modeled by a cubic regression on Legendre polynomials of animal age at calving, were included as fixed effects. The additive genetic and permanent environmental effects were modeled by Legendre polynomials on age at calving in months as basis functions, from second to fourth order. To define the most adequate structure to model the residual variance, the analyses started with a model considering equal number of regressions for all effects, second-order Legendre polynomial, and the polynomial order for additive genetic and animal permanent environmental effects were afterwards increased. The matrix representation of the RRM models is:

$$
\mathrm{y}=\mathrm{Xb}+\mathrm{Za}+\mathrm{Wap}+\mathrm{e}
$$

where $y$ is the vector of observations, $b$ is the vector of fixed effects, $a$ is the vector of random coefficients for additive direct effects, $a p$ is the vector of random coefficients for animal permanent environment effects, $e$ is the vector of residual effects, and $X, Z$, and $W$ are the corresponding incidence matrices. The model is based on the following assumptions:

$$
\mathrm{E}\left[\begin{array}{l}
\mathbf{y} \\
\mathbf{a} \\
\mathbf{a p}
\end{array}\right]=\left[\begin{array}{l}
\mathbf{X b} \\
\mathbf{0} \\
\mathbf{0}
\end{array}\right] ; \mathrm{V}\left[\begin{array}{c}
\mathbf{a} \\
\mathbf{a p} \\
\mathbf{e}
\end{array}\right]=\left[\begin{array}{ccc}
\mathbf{K}_{\mathbf{a}} \otimes \mathbf{A} & \mathbf{0} & \mathbf{0} \\
\mathbf{0} & \mathbf{K}_{\mathrm{ap}} \otimes \mathbf{I} & \mathbf{0} \\
\mathbf{0} & \mathbf{0} & \mathbf{R}
\end{array}\right]
$$

where $K_{a}$ and $K_{a p}$ are (co)variance matrices between random regression coefficients for additive genetic and animal permanent environment effects, respectively, $A$ is the relationship matrix, $I$ is an identity matrix, $\otimes$ is the Kronecker product between matrices, and $R$ is a block diagonal matrix containing residual variances. Correlations between random regression coefficients for different effects were set to zero. 
The covariance components and genetic parameters were estimated by the restricted maximum likelihood method using the WOMBAT program (Meyer, 2006). Results from different RRM were compared by the Schwarz Bayesian information criterion (BIC) (Schwarz, 1978), and by inspecting the variance component and genetic parameter estimates. Here, $p$ denotes the number of parameters estimated, $N$ the sample size, $\mathrm{r}(\mathrm{X})$ the rank of the coefficient matrix of fixed effect in the model of analysis, and $\log L$ the REML maximum $\log$ likelihood. The information criterion is then given as:

$$
\mathrm{BIC}=-2 \log L+p \log [N-\mathrm{r}(\mathrm{X})]
$$

The RRM are referred to as: ka.kap.resy, where $k a=$ the order of the covariance function for additive genetic effect, kap = animal permanent environmental effect, and res = residual variances modeled by a step function with $y$ classes.

The sires breeding values were predicted for MY305 at 37, 52, 66, 79 and 94 months of age (calving age), by REP, MT, and RRM. Only the sires $(\mathrm{N}=119)$ with progeny in the data were considered. Spearman correlations between breeding values of sires and the percentage of common sires chosen for higher MY305 with REP, MT, and RRM models at different ages at calving were calculated considering two selection intensities of selected sires: 5 and $10 \%$.

\section{RESULTS AND DISCUSSION}

The corresponding mean, standard deviation, and variation coefficient for total milk yield (MY305) was $1789 \pm 686 \mathrm{~kg}$. The mean MY305 obtained was higher than those estimated by Vasconcellos and Tonhati (1998) and Tonhati et al. (2000b) also for Brazilian buffaloes. MY305 increased from 24 to 90 months of age, approximately, since only the most productive cows remained in the herd until 6 to 8 years of age (Figure 1). After this age, the mean MY305 showed high variation, probably, as a consequence of decrease in the number of records. The number of calving per cow was 2.37 with a calving interval of 498 days.

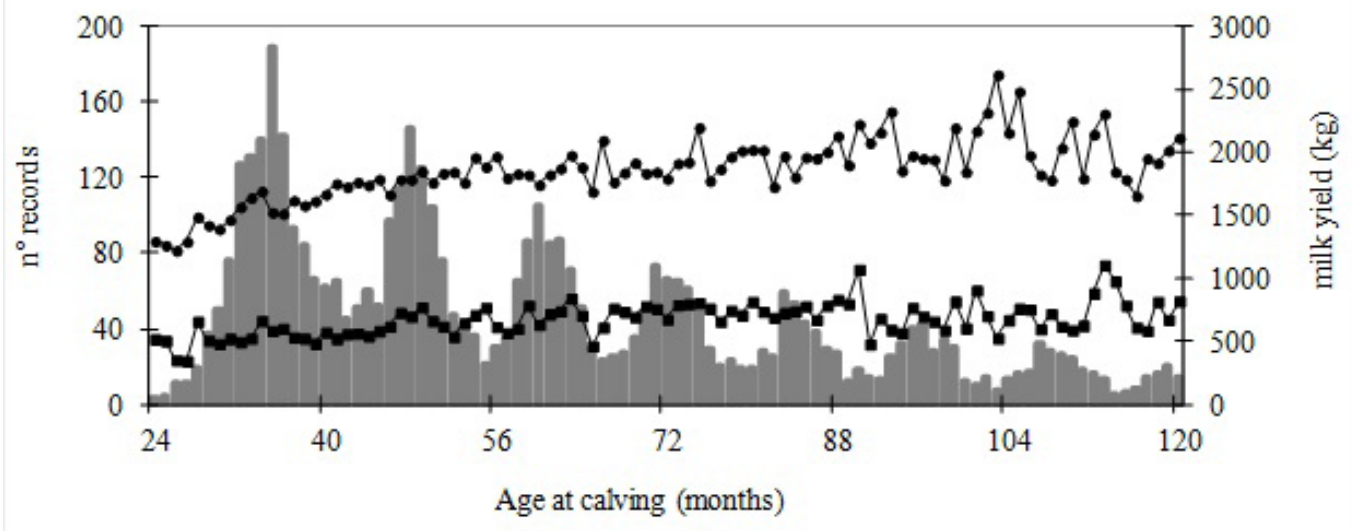

Figure 1. No. of animals and milk yield means (•) and standard deviations (๘). 
First, a model considering the same number of regressions for all effects was fitted, and the polynomial order for additive genetic and animal permanent environmental effects was then increased. A summary of RRM analyses with the maximum likelihood logarithmic function values ( $\log \mathrm{L})$ and Schwarz BIC are shown in Table 1. The model with heterogeneous residual variances showed a better fit than a model with homogenous residual variances (Table 2). These results indicated that residual variances had different behavior over the lactations, so it was necessary to consider a heterogeneous variance structure for the residual. According to $\log \mathrm{L}$ results, a model fitting the residual variance with a step function with 4 or 6 classes showed a similar fit. However, the BIC result showed that 4 residual classes were more adequate to model the residual variances. The statistical criterion BIC tests the lack of fit and the number of parameters estimated in the model, where models with lower BIC values are chosen as the best models to fit the data. Guo et al. (2002) working with milking records from the first to seventh parity of Danish Jersey cows and applying RRM assumed that residual variances were constant across parities. These results disagreed with those obtained in the present study.

\begin{tabular}{|c|c|c|c|c|c|c|}
\hline \multirow[b]{2}{*}{ Model } & \multicolumn{3}{|c|}{ Polynomial order } & \multirow[t]{2}{*}{$\mathrm{P}$} & \multirow[t]{2}{*}{$\log L$} & \multirow[t]{2}{*}{$\mathrm{BIC}$} \\
\hline & $\mathrm{k}$ & $\mathrm{k}_{\mathrm{n}}$ & $\mathrm{e}$ & & & \\
\hline L22.res1 & 2 & 2 & 1 & 7 & $-28,248.72$ & $56,555.98$ \\
\hline L22.res6 & 2 & 2 & 6 & 12 & $-28,228.82$ & $56,558.00$ \\
\hline L22.res4 & 2 & 2 & 3 & 10 & $-28,231.46$ & $56,546.56$ \\
\hline L23.res4 & 2 & 3 & 3 & 13 & $-28,215.79$ & $56,540.31$ \\
\hline L24.res4 & 2 & 4 & 3 & 17 & $-28,205.29$ & $56,552.76$ \\
\hline L32.res4 & 3 & 2 & 3 & 13 & $-28,217.63$ & $56,543.99$ \\
\hline L42.res4 & 4 & 2 & 3 & 17 & $-28,213.41$ & $56,569.02$ \\
\hline L33.res4 & 3 & 3 & 3 & 16 & $-28,210.97$ & $56,555.76$ \\
\hline L34.res4 & 3 & 4 & 3 & 20 & $-28,199.78$ & $56,566.84$ \\
\hline MT & & & & 30 & $-23,282.74$ & $46,810.24$ \\
\hline REP & & & & 3 & $-28,318.82$ & $56,662.73$ \\
\hline
\end{tabular}

Polynomial order for additive genetic $\left(\mathrm{k}_{\mathrm{a}}\right)$ and permanent environmental effect $\left(\mathrm{k}_{\mathrm{p}}\right)$, residual $(\mathrm{e})$ with heterogeneous (het) and homogeneous classes (hom), number of parameters (P), maximum likelihood logarithmic function values (Log L) and Schwarz Bayesian Information criterion (BIC).

Table 2. Genetic correlation estimates obtained by L23.res4 model (above the diagonal) and by MT model (below the diagonal) between MY305 at different ages.

\begin{tabular}{lccccc}
\hline & \multicolumn{5}{c}{ Age at calving (months) } \\
\cline { 2 - 6 } & 37 & 52 & 66 & 79 & 94 \\
\hline 37 & - & 0.97 & 0.92 & 0.85 & 0.95 \\
67 \\
66 & 0.97 & - & 0.98 & 0.99 & 0.90 \\
79 & 0.92 & 0.99 & - & - & 0.96 \\
94 & 0.93 & 0.99 & 0.99 & 0.80 \\
\hline
\end{tabular}

After defining the most adequate structure to model the residual variance, the polynomial order for additive genetic and animal permanent environmental effects was increased. The $\log$ likelihood $(\log \mathrm{L})$ value slightly increased with the number of parameters in the model (Table 1). The resultant BIC values indicated the model L23.res4, with 13 parameters, as the best to fit the data. Increasing the order of fit to two and three for the direct additive genetic and animal permanent environmental effects, respectively, did not improve the criterion (BIC). 
Also, for the animal permanent environmental effects, convergence problems and eigenvalues close to zero occurred when polynomial order was increased from 3 to 4. Guo et al. (2002) also used a third-order Legendre polynomial to model the additive genetic and permanent environmental effects in Danish Jersey cows.

According to $\log \mathrm{L}$ and BIC criteria, the REP model showed worse fit than the most adequate RRM (L23.het3). Despite that the MT model had a better fit than the L23.res4 model, a larger number of parameters (30) needed to be estimated with the MT model. Moreover, the database used with the MT model is not the same as that used with RRM analyses, since only the MY305 records closer to 37, 52, 66, 79, and 94 months of age (calving age) were considered in the analyses, and thus, the $\log \mathrm{L}$ and BIC criteria of the MT model are not comparable with RRM.

The additive genetic variance estimates obtained with the L23.res4 model increased with age (Figure 2A), and were similar to those obtained with REP model until 56 months of age. Guo et al. (2002) applying RRM observed that additive variance estimates for MY305 were maximal at third parity and decreased thereafter. Additive genetic variance estimates obtained with MT model showed a similar trend as those observed with the L23.res4 model at 37, 52, 79, and 94 months of age, but at other ages, estimates obtained with MT were higher. The results obtained in the present study showed heterogeneity of variance between records from different lactations. This heterogeneity is taken into account by the MT and L23.res4 models.
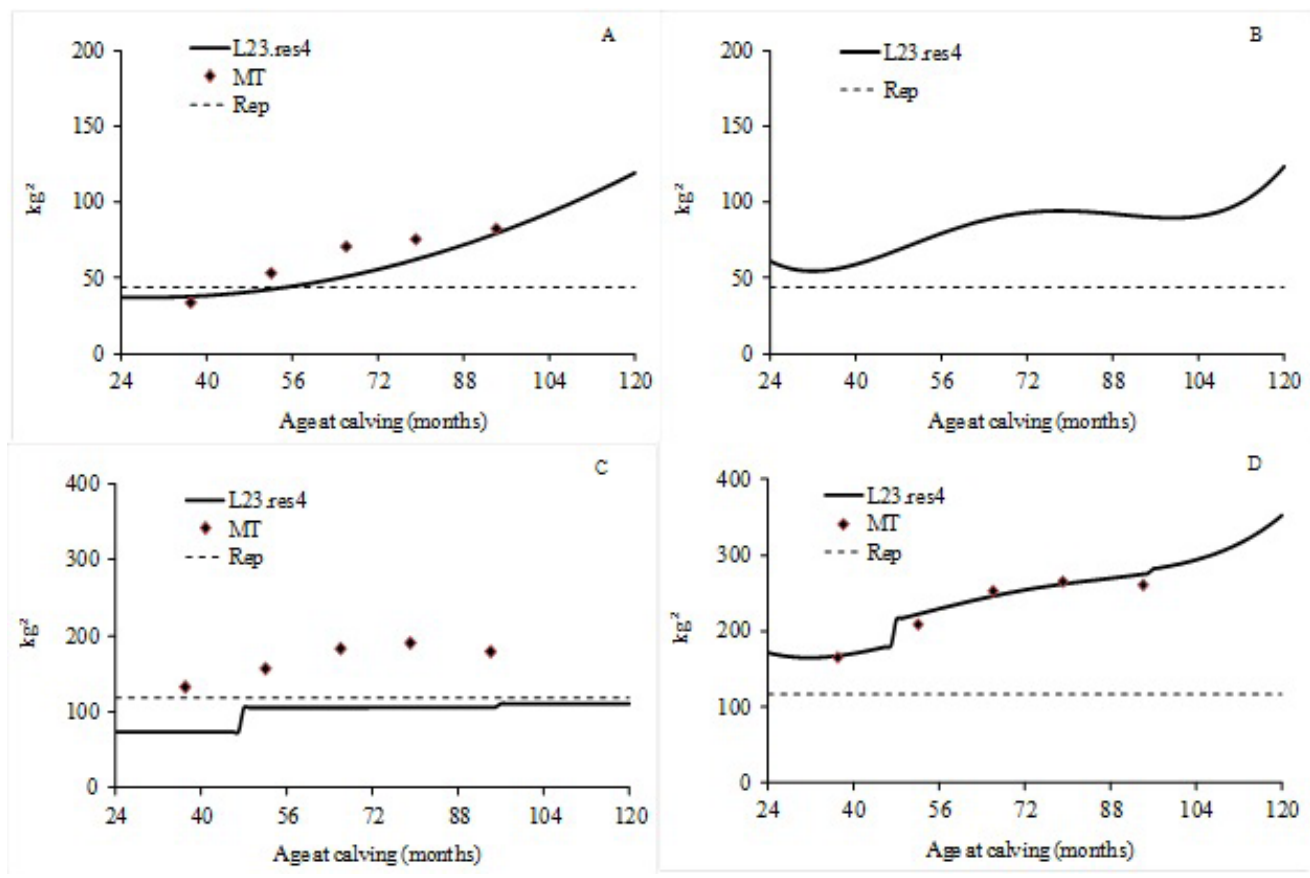

Figure 2. Additive genetic $\left(\mathbf{A} ; \sigma_{\mathrm{a}}^{2} / 1000\right)$, animal permanent environmental $\left(\mathbf{B} ; \sigma_{\text {ap }}^{2} / 1000\right)$ Phenotypic $\left(\mathbf{C} ; \sigma^{2} / 1000\right)$ and residual ( $\mathbf{D} ; \sigma_{\mathrm{e}}^{2} / 1000$ for RRM and repeatability model or $\sigma^{2}{ }_{\mathrm{e}+} \sigma_{\text {ap }}^{2}$ for multi-trait model) variance estimates for MY305 obtained with repeatability (REP), multi-trait (MT) and the best random regression model fitted (L23.res4). 
The additive genetic variance estimates obtained with MT increased until 66 months of age. This behavior is probably explained by the decrease in the number of records after 5 years of age. According to Meyer et al. (2004), multivariate analyses involving more than 3 or 4 traits are notorious for producing erratic estimates on occasion, particularly where the estimation procedure converges at the boundary of the parameter space. The animal permanent environmental variance estimates obtained with L23.res4 increased with age, and they were higher than that obtained with REP (Figure 2B).

For the MT and L23.res4 models, the residual variance estimates increased with age (Figure 2C). MT showed higher residual variances than did REP and L23.res4. The residual variance estimates obtained by L23.res4 were lower at early and intermediate ages than those obtained with MT and REP. Espinoza et al. (2007), working with Holstein milk yield records of the first four lactations, applied one-trait and MT models to estimate genetic parameters for MY305 and reported larger residual variance for later lactations.

The phenotypic, additive genetic, animal permanent environmental and residual variance estimates for MY305 obtained with the REP, MT, and L23.res4 models, are presented in Figure 2. Phenotypic variance estimates obtained with L23.res4 and MT were similar and increased with age (Figure 2D). Guo et al. (2002) reported that the phenotypic and permanent environmental variance estimates for MY305 obtained by RRM increased from lactation 1 to 7. The phenotypic variance estimates obtained with the REP were lower than with L23. res4 and MT.

For all models, the heritability estimates showed the same trend as those observed for additive genetic variance estimates. The MY305 heritability estimate obtained with REP was 0.20 (Figure 3A). The REP model has been extensively applied in dairy cattle to estimate genetic parameters for MY305 over multiple parities (Suzuki and VanVleck, 1994; Ferreira and Fernández, 2000; Espinoza et al., 2007). The heritabilities estimated with L23.res4 increased over ages, varying from 0.19 to 0.34 , and were slightly higher than that obtained with REP. These results disagreed with those reported by Guo et al. (2002), who observed that MY305 heritability estimates decreased over lactations. Araújo et al. (2007) applied RRM to estimate genetic parameters for MY305 in dairy buffaloes and dairy cattle, respectively, and reported that heritability increased until 12 to 14 years of age and then decreased.
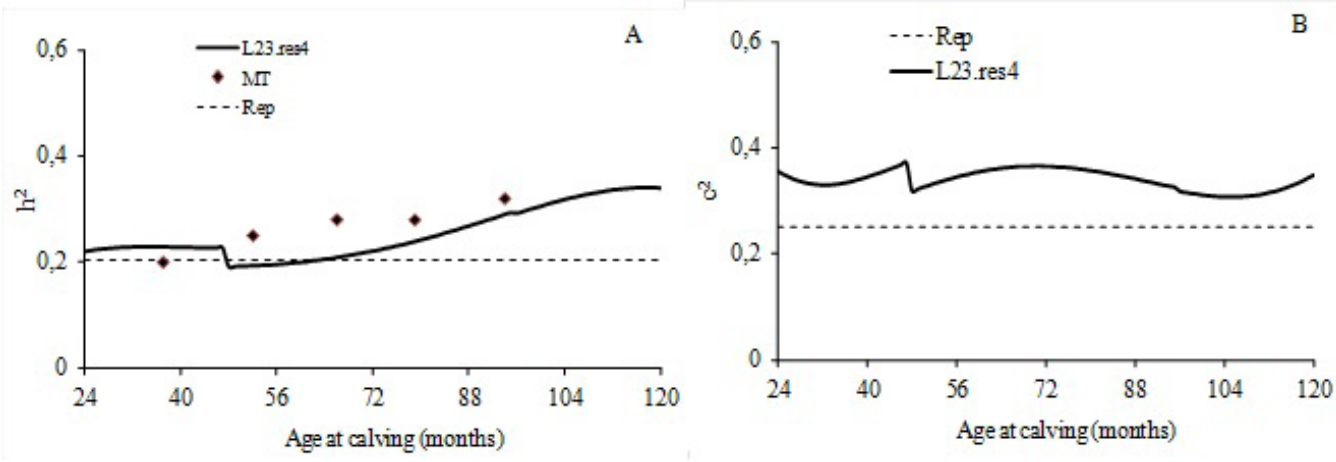

Figure 3. Heritability estimates A. and animal variance estimates B. as proportions of phenotypic variances for MY305 at different ages obtained with repeatability (REP), multi-trait (MT) and random regression model (L23.res4). 
For the MT model, heritability estimates ranged from 0.20 (37 months of age) to 0.32 (94 months of age) (Figure 3A). The heritability estimates obtained with MT after 37 months of age, were higher than those estimated with L23.res4. Heritability estimates applying MT models in dairy buffaloes are scarce in the literature (Mourad and Mohamed, 1995). In general, as the number of records decreased after 4 years, the heritability estimates obtained with MT were almost constant. In dairy cattle, several studies have reported (co)variance components and genetic parameter estimates for milk yield from multi-trait analyses, but only for the first three lactations (Meyer, 1984; Swalve and Van Vleck, 1987; Teepker and Swalve, 1988; Albuquerque et al., 1996). These studies have reported constant heritabilities over the first parities and high genetic correlations.

The animal variance estimates as proportions of phenotypic variances $\left(\mathrm{c}^{2}\right)$ obtained by RRM (L23.res4) were higher at extreme and intermediate ages (Figure 3B). Nevertheless, Guo et al. (2002) reported that animal variance estimates as proportions of phenotypic variances estimates obtained by RRM increased over parities.

The genetic correlation estimates for MY305 obtained by RRM (L23.res4) and MT models were very similar, and varied from 0.78 to 0.99 and from 0.78 to 0.99 , respectively (Table 2). Genetic correlation estimates between MY305 at first lactation (37 months of age) with MY305 at consecutive lactations obtained with L23.res4 and MT were almost similar. For lactations after 4 years of age, the genetic correlation estimated with L23.res4 was high, close to unity.

According to Teepker and Swalve (1988), Albuquerque et al. (1996) and Guo et al. (2002), the first lactation is a good indicator of the productive performance of a cow, since the genes that affect milk yield in the first lactation also influence yield in the subsequent lactations. The results obtained in the present study pointed out that selecting for higher total milk yield at first lactation will probably increase the total milk yield at consecutive lactations. Similar conclusions were drawn by Guo et al. (2002), with Danish Jersey cows and also applying RRM. However, Araújo et al. (2007), using RRM to adjust the MY305 in buffaloes over multiple parities, reported low and negative genetic correlations between the first total milk yield lactation with total milk yield at advanced ages.

Despite that some genetic correlation estimates were high and close to unity between adjacent ages, genetic correlations different from unity demonstrates a need to consider them as different traits, mainly between MY305 at early (37 months of ages) with later ages (94 months of age), and REP could be inappropriate for modeling this type of trait. Moreover, the heterogeneity of variances between lactations shown in Figure 2 must be taken into account. According to Swalve and VanVleck (1987), the REP model can be justified by the computational requirements of MT models only if the genetic correlations are close to one.

Considering all sires, the rank correlation between breeding values for MY305 at different ages predicted by REP, MT, and L33.het3 were high (Table 3). The sire breeding value rank correlations between the REP and MT models were lower than those obtained for rank correlations between the REP and L23.res4 models. With high selection intensities, the results indicated that practically the same sires would be selected by REP and L23.res4. On the other hand, different sires would be selected if a MT were used instead of a REP model. Differences in sire classification between MT and REP are expected since both models made different assumptions and gave different (co)variance component estimates. 
Table 3. Spearman correlations applying different selection intensities and number of sires selected for higher MY305 based on repeatability model (RP), that would also be selected based on breeding values predicted by multi-trait (MT) or L23.res4 ${ }^{\text {a }}$.

\begin{tabular}{|c|c|c|c|}
\hline$\%$ of sires selected on RP model & No. of sires selected on RP model & No. of sires selected on MT model & No. of sires selected on L23.res 4 \\
\hline \multicolumn{4}{|l|}{ Age at calving (37 months) } \\
\hline 5 & 6 & $5\left(0.60^{b}\right)$ & $6(0.89)$ \\
\hline 10 & 12 & $11(0.63)$ & $11(0.89)$ \\
\hline 100 & 119 & $119(0.92)$ & $119(0.97)$ \\
\hline \multicolumn{4}{|l|}{ Age at calving (52 months) } \\
\hline 5 & 6 & $5(0.60)$ & $6(0.89)$ \\
\hline 10 & 13 & $10(0.67)$ & $11(0.89)$ \\
\hline 100 & 119 & $119(0.91)$ & $119(0.97)$ \\
\hline \multicolumn{4}{|l|}{ Age at calving ( 66 months) } \\
\hline 5 & 6 & $5(0.60)$ & $6(0.89)$ \\
\hline 10 & 13 & $10(0.67)$ & $11(0.89)$ \\
\hline 100 & 119 & $119(0.91)$ & $119(0.97)$ \\
\hline \multicolumn{4}{|l|}{ Age at calving ( 79 months) } \\
\hline 5 & 6 & $5(0.60)$ & $6(0.89)$ \\
\hline 10 & 13 & $10(0.67)$ & $11(0.89)$ \\
\hline & 119 & $119(0.91)$ & $119(0.97)$ \\
\hline \multicolumn{4}{|l|}{ Age at calving (94 months) } \\
\hline 5 & 6 & $5(0.60)$ & $6(0.89)$ \\
\hline 10 & 13 & $10(0.80)$ & $11(0.89)$ \\
\hline 100 & 199 & $119(0.89)$ & $119(0.97)$ \\
\hline
\end{tabular}

a 2 23.res4 corresponding to the order of $\mathrm{k}_{\mathrm{a}}(2)$ and $\mathrm{k}_{\mathrm{pe}}(3)$ effects and to the residual variance structure modeled by a step function (het) assuming 4 variance classes; ${ }^{\mathrm{b}}$ Rank correlation between breeding values predicted by RP model with MT or L23.res4 model.

The sire breeding value rank correlations between the L23.res4 and MT models followed the same trend as those obtained between REP and L23.res4 (results not shown). As the selection intensity increased, the sire rank correlations and the number of common sires selected by L23.res4 and MT decreased.

Even though the statistical criteria pointed to MT as the most suitable model to fit the data, L23.res3 is a more parsimonious model, which is less computation demanding and time consuming. These aspects are very important to define an appropriate model for genetic evaluation on a large scale. In the present study, with a small data set, little differences in time demand (few hours) were observed between the MT and L23.res3 models, but greater differences are expected with a large data set, such as in national genetic evaluations. Also, L23. res3 allows a better fitting of environmental and genetic effects, since lower residual variance estimates are obtained with this model. In this sense, several studies with simulated and real data (Strabel et al., 2001; Tier and Meyer, 2004; Boligon et al., 2011) have confirmed that RRM provide (co)variance components with lower prediction error variance and more reliable breeding value accuracy estimates than finite models (MT models). Finally, model selection is not an easy task in animal breeding. As stated by Box (1976), all models are wrong but some are useful. Hence, other studies are necessary to evaluate the predictive ability of models chosen as most appropriate for genetic evaluation. In this sense, one possibility is the implementation of cross-validation or bootstrap methods or evaluation of the selected models in other large data sets.

In conclusion, genetic correlations for milk yield between ages are not equal to unity showing that the estimation of genetic parameters for milk yield using a RMM on age at calving was more reasonable than an animal REP model. Applying RRM to model MY305 over 
multiple ages, the residual variances should be modeled through heterogeneous classes, with four classes of residual variances being the most adequate. It seems that a linear and quadratic Legendre polynomial to model the additive genetic and animal permanent environmental effects, respectively, may be sufficient to explain more parsimoniously the changes in MY305 genetic variation over ages. Random regression analyses on Legendre polynomial of age at calving are able to describe the genetic variability for total milk yield at different ages and would be most appropriate for genetic evaluation of dairy buffaloes.

\section{REFERENCES}

Albuquerque LG, Keown JF and Van Vleck LD (1996). Genetic parameters of milk, fat, and protein yields in the first three lactations, using an animal model and restricted maximum likelihood. Braz. J. Genet. 19: 79-86.

Araújo CV, Ramos AA, Araújo SI, Chaves LC, et al. (2007). Buffalos milk yield analysis using random regression models. In: world buffalo congress, 12, 2007. Ital. J. Anim. Sci. 6 (Suppl 2): 279-282.

Bignardi AB, El Faro L, Cardoso VL, Machado PF, et al. (2009). Random regression models to estimate test-day milk yield genetic parameters Holstein cows in Southeastern Brazil. Livest. Sci. 123: 1-7.

Boligon AA, Baldi F, Mercadante ME, Lobo RB, et al. (2011). Breeding value accuracy estimates for growth traits using random regression and multi-trait models in Nelore cattle. Genet. Mol. Res. 10: 1227-1236.

Box GEP (1976). Science and statistics. J. Am. Stat. Assoc.71: 791-799.

Breda FC, Albuquerque LG, Euclydes RF, Bignardi AB, et al. (2010). Estimation of genetic parameters for milk yield in Murrah buffaloes by Bayesian inference. J. Dairy Sci. 93: 784-791.

Espinoza AP, Villavicencio JLE, González-Peña D, Iglesias DG, et al. (2007). Estimation of covariance components for the first four lactations in Holstein cattle according to different models. Zootecnia Trop. 25: 9-18.

Ferreira GB and Fernández HD (2000). Genetic parameters for productive traits in Holstein cows in Goias state, Brazil. Rev. Braz. Zootec. 29: 421-426.

Freitas AF, Durães MC, Valente J, Teixeira NM, et al. (2001). Genetic parameters of milk and fat yields of Holstein cows in the three first lactations. Braz. J. Anim. Sci. 30: 709-713.

García-Cortés LA, Moreno L, Varona L and Rico M (1995). (Co) variance component estimation of yield traits between different lactations using animal model. Livest. Prod. Sci. 43: 111-117.

Guo Z, Lund MS, Madsen P, Korsgaard I, et al. (2002). Genetic parameter estimation for milk yield over multiple parities and various lengths of lactation in Danish Jerseys by random regression models. J. Dairy Sci. 85: 1596-1606.

Meyer K (1984). Estimates of genetic parameters for milk and fat yield for first three lactations in British friesian cows. Anim. Prod. 38: 313-322.

Meyer K (1998). Estimating covariance functions for longitudinal data using a random regression model. Genet. Sel. Evol. 30: 221-240.

Meyer K (2004). Scope for a random regression model in genetic evaluation of beef cattle for growth. Livest. Prod. Sci. 86: 69-83.

Meyer K (2006). "WOMBAT" - Digging Deep for Quantitative Genetic Analyses by Restricted Maximum Likelihood. Proc. 8th World Congr. Genet. Appl. Livest. Prod, CD-ROM., Belo Horizonte.

Meyer K and Hill WG (1997). Estimation of genetic and phenotypic covariance functions for longitudinal data by restricted maximum likelihood. Livest. Prod. Sci. 47: 185-200.

Meyer K, Johnston DJ and Graser HU (2004). Estimates of the complete genetic covariance matrix for traits in multi-trait genetic evaluation of Australian Hereford cattle. Aust. J. Agri. Res. 55: 195-210.

Mourad KA and Mohamed MM (1995). Genetic and phenotypic aspects of milk yield traits and reproductive performance of Egyptian buffaloes. Egypt. J. Anim. Prod. 32: 125-137.

Powell RL and Norman HD (2006). Major advances in genetic evaluation techniques. J. Dairy Sci. 89: 1337-1348.

Rosati A and Van Vleck LD (2002). Estimation of genetic parameters for milk, fat, protein and mozzarella cheese production for the Italian river buffalo Bubalus bubalis population. Livest. Prod. Sci. 74: 185-190.

Schaeffer LR (2004). Application of random regression models in animal breeding. Livest. Prod. Sci. 86: 35-45.

Schwarz G (1978). Estimating the dimension of a model. Ann. Stat. 6: 461-464.

Sesana RC, Bignardi AB, Borquis BR, El Faro L, et al. (2010). Random regression models to estimate genetic parameters for test-day milk yield in Brazilian Murrah buffaloes. J. Anim. Breed. Genet. 127: 369-376.

Strabel T, Misztal I and Bertrand JK (2001). Approximation of reliabilities for multiple-trait model with maternal effects. J. Anim. Sci. 79: 833-839. 
Suzuki M and Van Vleck LD (1994). Heritability and repeatability for milk production traits of Japanese Holsteins from an animal model. J. Dairy Sci. 77: 583-588.

Swalve HH and Van Vleck LD (1987). Estimation of genetic (co)variance for milk yields in first three lactations using an animal model and restricted maximum likelihood. J. Dairy Sci. 70: 842-849.

Teepker G and Swalve HH (1988). Estimation of genetic parameters for milk production in the first lactations. Livest. Prod. Sci. 20: 193-202.

Tier B and Meyer K (2004). Approximating prediction error covariances among additive genetic effects within animals in multiple-trait and random regression models. J. Anim. Breed. Genet.121: 77-89.

Tonhati H, Munõz MFC, Oliveira JA, Duarte JMC, et al. (2000a). Genetic parameters of milk production, fat and protein contents in buffalo milk, Braz. Braz. J. Anim. Sci. 29: 2051-2056.

Tonhati H, Vasconcellos FB and Albuquerque LG (2000b). Genetic aspects of productive and reproductive traits in a murrah buffalo herd in São Paulo, Brasil. J. Anim. Breed. Genet. 117: 331-336.

Tonhati H, Cerón-Muñoz MF, Oliveira JA, El Faro L, et al. (2008). Test-day milk yield as a selection criterion for dairy buffaloes (Bubalus bubalis Artiodactyla, Bovidae). Genet. Mol. Biol. 31 -: 674-679.

Vasconcellos BF and Tonhati H (1998). Inbreeding and Its effects on some productive and reproductive traits in a Murrah Buffalo herd. J. Anim. Breed. Genet. 115: 299-306. 